\title{
Stability of unicortical locked fixation versus bicortical non-locked fixation for forearm fractures
}

\author{
Timothy J Pater ${ }^{1}$, Steve I Grindel ${ }^{1}$, Gregory J Schmeling ${ }^{1,2}$ and Mei Wang ${ }^{1,2}$
}

Locking plate fixation is being widely applied for fixation of forearm fractures and has many potential advantages, such as fixed angle fixation and improved construct stability, especially in osteoporotic bone. Biomechanical data comparing locking devices to commonly used Low Contact Dynamic Compression (LCDCP) plates for the fixation of forearm fractures has been lacking. The purpose of this study was to compare the fixation stability of a 3.5-mm unicortical locked plate with bicortical non-locked LCDCP plates. Six matched pairs of fresh frozen cadaveric forearms were randomly assigned to unicortical locked and bicortical unlocked groups. Non-destructive four-point bending and torsional test was performed on the ulna and radius separately, using a servohydraulic testing system to obtain construct stiffness of the intact specimens and specimens after osteotomy and plating. The specimens were then loaded to failure to test the fixation strength. The locked unicortical fixation showed significantly higher bending stiffness than the unlocked bicortical fixation, but with significantly lower stiffness and strength in torsion. Fixation strength was comparable between the two groups under bending, but significantly greater in the bicortical non-locked group under torsion. Findings from this study suggest that postoperative rehabilitation protocols may need modification to limit torsional loading in the early stage when using locked unicortical fixation. The study also points out the potential advantage of a hybrid fixation that combines locked unicortical and unlocked bicortical screws.

Bone Research (2014) 2, 14014; doi:10.1038/boneres.2014.14; published online 1 July 2014

\section{INTRODUCTION}

Open reduction and internal fixation of forearm fractures are among the most commonly performed orthopedic procedures. Biomechanical analysis of traditional forearm compression plates has been performed in the cadaveric model. Cortical fixation in various constructs influences fixation stability. The number of cortices that fixation screws engage is important to construct stability. ${ }^{1-2}$ However, fixation involving a larger number of cortices may weaken bone following plate removal. Studies have shown that screw holes weaken cortical bone and are potential sites for refracture. ${ }^{3}$

Locking plate fixation is now being clinically employed for fixation of forearm fractures and has many theoretical advantages. ${ }^{4-6}$ The potential advantages include fixed angle fixation, improved construct stability, especially in osteoporotic bone and the ability to use locked unicortical screws potentially decreasing the risk of refracture following plate removal. Biomechanical data comparing locking devices to commonly used Low Contact Dynamic Compression (LCDCP) plates for the fixation of forearm fractures have not been established.

The purpose of this study was to determine the mechanical behavior of 3.5-mm locking plates compared to LCDCP plates. The study attempts to answer the clinical question of whether it is more beneficial to fix a fractured forearm with bicortical screws or unicortical locked screws from the perspectives of fixation stability.

\section{MATERIALS AND METHODS}

Six matched pairs of fresh frozen cadaveric forearms were procured (donor mean age: 83 years, three male and three female), and were completely dissected from their soft tissues. Prior to testing, plain radiographs of all specimens were taken to rule out any underlying osseous pathology. Bone densiometry scanning [dual energy X-ray absorptiometry (DEXA) scan] was performed on each specimen. The purpose of DEXA scanning was to rule out

'Department of Orthopaedic Surgery, Medical College of Wisconsin, Milwaukee, WI, USA and ${ }^{2}$ Orthopaedic \& Rehabilitation Engineering Center, Marquette University, Milwaukee, WI, USA

Correspondence: M Wang (meiwang@mcw.edu)

Received: 28 March 2014; Revised: 18 April 2014; Accepted: 22 April 2014 
significant side-to-side bone density differences related to hand dominance.

\section{Bending test}

One ulna in each pair was randomly selected to receive either locked unicortical or non-locked bicortical plate fixation. Bicortical fixation was performed using eight-hole, 3.5-mm LCDCP (Synthes, Paoli, PA, USA). Locked unicortical fixation was performed using eight-hole $3.5-\mathrm{mm}$ locking compression plates (Synthes). Screws were placed in the three most proximal and three most distal holes of each plate, leaving the center two holes open.

Each specimen was potted in customized jigs with dental cement and underwent a series of four-point bending tests using a servohydraulic, bimodal materials testing system (Model 809; MTS Systems, Eden Prairie, MN, USA). Fourpoint bending tests were performed in the apex dorsal plane (plate acted as tension band) under a displacement control (Figure 1). Three cycles of preconditioning were preformed for all tests.

First, a non-destructive four-point bending test was performed on the intact specimens that were predrilled to receive either locking unicortical or unlocking bicortical screws. The maximum moment applied was $7.5 \mathrm{~N} \cdot \mathrm{m}$ and the loading rate was $0.1 \mathrm{~mm} \cdot \mathrm{s}^{-1}$. This peak moment was selected to simulate physiological loading of the forearm, and was scaled down by $25 \%-50 \%$ from the published bending strength of the intact radius. ${ }^{7}$ The compressive force and corresponding vertical displacement measured from the MTS built-in load cell and displacement sensor were converted into the applied bending moment and corresponding angular displacement. Bending stiffness of the ulna was obtained from the linear region of the load-displacement plot. Next, an osteotomy was performed on the intact specimen using hand saw in the middle of the bone, and the plates were instrumented. Nondestructive four-point bending tests were repeated to

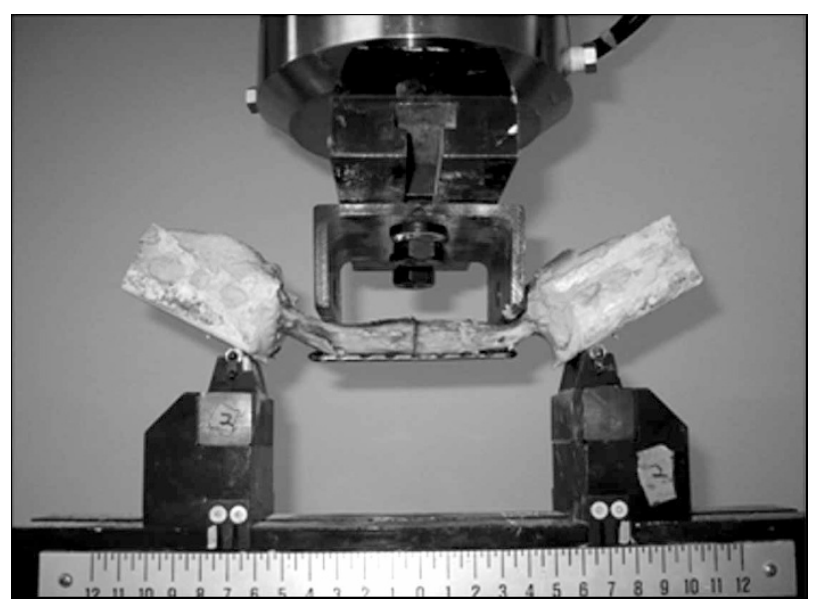

Figure 1. Experimental set-up for four-point bending test. evaluate bending stability of the plated construct. Finally, the plated ulna construct was loaded to failure at the same loading rate to determine the bending strength of the plated construct. Construct failure was defined as the first drop in load, and the maximum compressive force at the point of failure was recorded.

\section{Torsional test}

Matched pairs of radii from the forearm specimens were prepared in identical fashion. This portion of the study was designed to evaluate torsional construct stability. Specimens were potted in customized jigs (Figure 2). Torsional load up to $4 \mathrm{~N} \cdot \mathrm{m}$ was applied to intact specimens (with screw holes prepared) under displacement control. The peak torque was scaled down by $25 \%$ of the ultimate torques of the intact radius and ulna reported by Yamada. $^{8}$ The tests were repeated following osteotomy with the plate applied, and the maximum torsion applied was reduced to $2 \mathrm{~N} \cdot \mathrm{m}$ to avoid damaging the weakened construct. Finally, the plated radial construct was loaded to failure in torsion.

\section{Data analysis}

To evaluate fixation stability between the plated constructs from the locking unicortical and bicortical LCDCP groups, bending and torsional stiffness were compared using paired, one-tailed t-test. For comparison of the fixation strength, the differences in maximum bending and torsional loads between the two groups were also compared with the paired t-test. In addition, bending and torsional stiffness of the intact specimens with unicortical screw holes and those with bicortical screw holes were compared as an indication for potential for refracture risk. Significant level of $P<0.05$ was used for all tests. Finally, to determine the effect of bone density on the construct behaviors, Pearson correlation coefficients were determined between the DEXA bone mineral density measurements

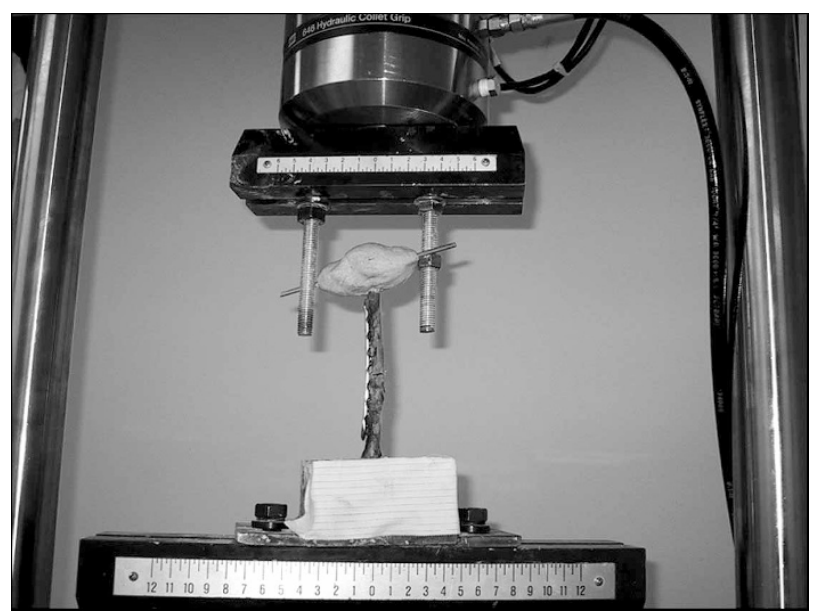

Figure 2. Experimental set-up for torsional test. 
and the stiffness or strength of the construct. A $P$-value of less than 0.05 was considered significant.

\section{RESULTS}

Bending

Intact ulnae with unicortical screw holes showed an average increased stiffness of $19 \%$ compared to intact ulnae with bicortial screw holes $\left(327 \mathrm{~N} \cdot \mathrm{mm}^{-1}\right.$ versus $275 \mathrm{~N} \cdot \mathrm{mm}^{-1}$, $P<0.03)$. Osteotomized specimens that were reconstructed using unicortical locked plates showed an average increased stiffness of $21 \%$ when compared to those specimens plated using bicortical fixation $\left(185 \mathrm{~N} \cdot \mathrm{mm}^{-1}\right.$ versus $\left.153 \mathrm{~N} \cdot \mathrm{mm}^{-1}, P<0.03\right)$. There was no difference in average failure load of unicortical locked plate fixation compared to bicortical non-locked plate fixation (1 $285 \mathrm{~N}$ versus $1289 \mathrm{~N}, P<0.49$ ). Detailed results are listed in Table 1.

Torsion

Intact radii with unicortical screw holes showed an insignificant $3.3 \%$ increase in stiffness when compared to intact radii with bicortical screw holes $(0.64 \mathrm{~N} \cdot \mathrm{m}$ per degree versus $0.6 \mathrm{~N} \cdot \mathrm{m}$ per degree, $P=0.33$ ). The mean stiffness of osteotomized specimens plated with unicortical locked fixation showed a decrease of $44 \%$ when compared to specimens plated with bicortical non-locked fixation $(0.12 \mathrm{~N} \cdot \mathrm{m}$ per degree versus $0.21 \mathrm{~N} \cdot \mathrm{m}$ per degree, $P<0.013)$. Maximum torque at failure for unicortical locked fixation was $59 \%$ less than specimens plated with bicortical non-locked plate fixation $(2.67 \mathrm{~N} \cdot \mathrm{m}$ versus $6.57 \mathrm{~N} \cdot \mathrm{m}, P<0.002)$. Detailed results are listed in Table 2.

\section{Effect of bone density}

Bone densitometry testing as measured by DEXA scanning showed no significant side to side differences within each matched pair. There was a moderate relationship between bone density and bending $(R=0.75, P=0.09)$ and torsional $(R=0.88, P=0.02)$ stiffness of the intact specimens from the unicortical group. The bone density also had significant influence on the bending and torsional strength $(R=0.89, P=0.02)$ of the unicortically plated constructs.
There was no statistically significant relationship between bone density and stiffness of either plated constructs.

\section{DISCUSSION}

Traditional closed treatment methods for adult forearm fractures such as casting and bracing have long ago given way to operative intervention. Fixation of forearm fractures are among the most often performed procedures in orthopedics today. Because forearm plating is performed so commonly, it is important to consider the biomechanical properties of implants to appropriately apply them in patients.

Our study focuses on evaluating differences in fixation behavior of locked plates and LCDCP plates and delineating potential advantages of each. LCDCP plating with rigid fixation has been employed with success for many years and is widely accepted by orthopedic surgeons in the treatment of forearm fractures. The use of these implants generally involves standard AO technique with anatomic reduction, compression and rigid fixation. It is commonly accepted that adequate fixation involves six to eight cortices of screw fixation above and below the fracture site when using these implants.

Biomechanical studies comparing stability of new generation locked plating systems to that of traditional implants have been few. Marti et al., ${ }^{10}$ in a biomechanical study, compared locked Less Invasive Stabilization System plating to Dynamic Condylar Screw and Condylar Buttress Plating in the femur in a cadaver model. The results suggest enhanced ability to withstand high loads when using unicortical locked fixation when compared to traditional implants. Although biomechanical studies have been performed in forearm models comparing a variety of implants and constructs, no studies to date have focused on the use of locked implants in the forearm. ${ }^{1-14}$

Recently, 3.5-mm locking compression plates have been made available for clinical use (Synthes). These plates are based on LCDCP designs, but offer the option of locked fixation and the theoretical advantages of fixed angle fixation with improved construct stability, improved

Table 1. Mean (s.d.) results from the four-point bending tests on the matched ulna specimens

\begin{tabular}{|c|c|c|c|c|}
\hline & Bicortical & Unicortical & \% Difference Unicortical vs. bicortical & $P$-value \\
\hline Intact stiffness $/\left(\mathrm{N} \cdot \mathrm{mm}^{-1}\right)$ & $274.8 \pm 136.4$ & $327.1 \pm 133.0$ & $+19 \%$ & 0.03 \\
\hline Reconstructed stiffness/(N. $\left.\mathrm{mm}^{-1}\right)$ & $153.0 \pm 55.3$ & $185.0 \pm 75.0$ & $+21 \%$ & 0.03 \\
\hline Reconstructed strength/N & $1285.0 \pm 628.0$ & $1289.0 \pm 468.0$ & $+0.3 \%$ & 0.49 \\
\hline
\end{tabular}

Table 2. Mean (s.d.) results from the torsional tests on the matched radial specimens

\begin{tabular}{lcccc}
\hline & & & \% Difference \\
& Bicortical & Unicortical & Unicortical vs. bicortical & $P$-value \\
\hline Intact stiffness/(N.m per degree) & $0.62 \pm 0.29$ & $0.64 \pm 0.26$ & $+3.3 \%$ & 0.330 \\
Reconstructed stiffness/(N.m per degree) & $0.21 \pm 0.05$ & $0.12 \pm 0.07$ & $-43.8 \%$ & 0.010 \\
Reconstructed strength/(N.m) & $6.57 \pm 2.39$ & $2.67 \pm 0.61$ & $-59.4 \%$ & 0.002 \\
\hline
\end{tabular}


preservation of biology, improved fixation in osteoporotic bone and less risk of refracture following plate removal. Several authors have reported a significant incidence of refracture after plate removal with traditional forearm fixation. ${ }^{15-16}$ The ability to use locked unicortical screws theoretically lowers the risk of refracture following plate removal. In our cadaveric forearm model, intact specimens with unicortical screw holes were stiffer during the application of bending and torsional loads than those with bicortical screw holes. This suggests an advantage of unicortical locked plate fixation in the treatment of forearm fractures in that immediately after hardware removal, the bone would be better able to resist four-point bending and torsional loads. This implies that fracture or refracture upon hardware removal is less likely when using unicortical locked fixation.

An additional proposed advantage of locked cortical fixation is improved construct stability. In our model, unicortical locked plating of the osteotomized specimens showed increased stiffness during four-point bending. Comparison of the unicortical locked and bicortical nonlocked groups showed equivalent strength with regard to load to failure in four point bending. This suggests a potential advantage of unicortical locked plating in that it is stronger in four-point bending under normal physiological loads.

However, specimens fixed with unicortical locked plating showed decreased stiffness during torsional loading when compared to bicortical LCDCP plate fixation of the osteotomized specimens. Similarly, unicortical locked plating of the osteotomized radii specimens had a much smaller torque to failure when compared to bicortical nonlocked plating of the osteotomized specimens. This is a potential disadvantage of unicortical locked plate fixation in the treatment of forearm fractures in that it is more likely to fail during torsional loads. This suggests that postoperative rehabilitation protocols may need to be modified to avoid torsional loading when unicortical locked plate fixation is used in the treatment of forearm fractures.

There are some limitations to the study model. The model used in this study is a single bone forearm model. Although the single bone model is commonly accepted in the literature as a means of testing forearm construct stability, the fact remains that the forearm is a functionally complex two bone system with unique soft tissue contributions. One must take this into account when applying this data in the more complex in vivo forearm.

Furthermore, this study does not examine potential advantages and disadvantages of each device with regard to the biology of fracture healing. Fixed angle devices have the theoretical advantage of improved biology preservation and therefore, improved rates of healing compared to compression plating. A number of studies have examined the effect of traditional forearm constructs on the biology of fracture healing. ${ }^{1,2,17}$ Similarly, minimally invasive and less invasive reduction techniques with and without fixed angle devices have been studied clinically in the tibia and distal femur. ${ }^{17-20}$ These studies highlight increased attention in more recent years to the biology of fracture healing. Therefore, biomechanical stability cannot be considered in isolation when considering implant choice. The issue of biology was not addressed in this study and should be the subject of future study.

The bone density seemed to have no significant effect on the fixation stiffness of either the unicortical or bicortical group. However, for the unicortically plated construct, the bone density was a significant factor for both fixation strength and refracture risk. Future studies may focus on the relative value of locked fixation in osteoporotic bone in the specific case of forearm fractures.

In summary, unicortical locked fixation and bicortical non-locked fixation both appear to afford adequate construct stability for a forearm simple fracture model in the immediate postoperative stage. Unicortical locked fixation demonstrated greater stability against bending loads of the forearm, and potentially decreased refracture risk following plate removal. However, rehabilitation protocols may need to be modified when using this fixation to limit torsional loading in the early postoperative period.

\section{Conflict of interest}

The authors declare that they have no conflicts of interest.

\section{References}

1 Jain R, Podworny N, Hearn T et al. Effect of stainless steel and titanium low-contact dynamic compression plate application on the vascularity and mechanical properties of cortical bone after fracture. J Orthop Trauma 1997; 11: 490-495.

2 Jain R, Podworny N, Hupel TM et al. Influence of plate design on cortical bone perfusion and fracture healing in canine segmental tibial fractures. J Orthop Trauma 1999; 13: 178-186.

3 Perren SM, Cordey J, Rahn BA et al. Early temporary porosis of bone induced by internal fixation implants. A reaction to necrosis, not to stress protection? Clin Orthop Relat Res 1988; 232: 139-151.

4 Leung F, Chow SP. Locking compression plate in the treatment of forearm fractures: a prospective study. J Orthop Surg 2006; 14: 291-294.

5 Sommer C, GautierE, Muller M, Helfet DL, Wagner M. First clinical results of the Locking Compression Plate (LCP). Injury 2003; 34: B43-B54.

6 Miranda MA. Locking plate technology and its role in osteoporotic fractures. Injury 2007; 38: S35-S39.

7 Lochmuller E, Lill CA, Kuhn V, Schneider E, Eckstein F. Radius bone strength in bending, compression, and falling and its correlation with clinical densitometry at multiple sites. J Bone Mineral Res 2002; 17: 1629-1638.

8 Yamada H. Aging rate for the strength of human organs and tissues. In: Evans FG (ed.), Strength of biological materials. Baltimore: Williams \& Wilkins, 1970: 272-280.

9 Kellam JF, Jupiter JB. Diaphyseal fractures of the forearm. In: Browner B (ed.), Skeletal Trauma. Philadelphia, PA: WB Saunders, 1998: 1421-1454.

10 Marti A, Fankhauser C, Frenk A et al. Biomechanical evaluation of the less invasive stabilization system for the internal fixation of distal femur fractures. J Orthop Trauma 2001; 15: 482-487. 
11 ElMaraghy AW, ElMaraghy MW, Nousiainen M et al. Influence of the number of cortices on the stiffness of plate fixation of diaphyseal fractures. J Orthop Trauma 2001; 15: 186-191.

12 Jain R, Podworny N, Hearn $\mathrm{T}$ et al. A biomechanical evaluation of different plates for fixation of canine radial osteotomies. J Trauma 1998; 44: 193-197.

13 Jones DJ, Henley MB, Schemitsch EH et al. A biomechanical comparison of two methods of fixation of fractures of the forearm. J Orthop Trauma 1995; 9: 198-206.

14 Sanders R, Haidukewych GJ, Milne T et al. Minimal versus maximal plate fixation techniques of the ulna: the biomechanical effect of number of screws and plate length. J Orthop Trauma 2002; 16: 166-171.

15 Deluca PA, Lindsey RW, Ruwe PA. Refracture of bones of the forearm after the removal of compression plates. J Bone Joint Surg Am 1988; 70 1372-1376.

16 Langkamer VG, Ackroyd CE. Removal of forearm plates. A review of the complications. J Bone Joint Surg Am Br 1990; 72: 601-604.
17 Perren SM. Minimally invasive internal fixation history, essence and potential of a new approach. Injury 2001; 32: SA1-SA3.

18 Frigg R. Locking Compression Plate (LCP). An osteosynthesis plate based on the Dynamic Compression Plate and the Point Contact Fixator (PCFix). Injury 2001; 32: 63-66.

19 Frigg R, Appenzeller A, Christensen Retal. The development of the distal femur Less Invasive Stabilization System (LISS). Injury 2001; 32: S24S31.

20 Krettek C, Muller M, Miclau T. Evolution of minimally invasive plate osteosynthesis (MIPO) in the femur. Injury 2001; 32: S14-S23.

(c) (i) (-) This work is licensed under a Creative Commons AttributionNonCommercial-NoDerivs 3.0 Unported License. The images or other third party material in this article are included in the article's Creative Commons license, unless indicated otherwise in the credit line; if the material is not included under the Creative Commons license, users will need to obtain permission from the license holder to reproduce the material. To view a copy of this license, visit http://creativecommons.org/licenses/by-nc-nd/3.0/ 